Document downloaded from:

http://hdl.handle.net/10251/82923

This paper must be cited as:

Mira-Iglesias, A.; Conejero, JA.; Navarro Pardo, E. (2016). Natural visibility graphs for diagnosing attention deficit hyperactivity disorder (ADHD). Electronic Notes in Discrete Mathematics. 54:337-342. doi:10.1016/j.endm.2016.09.058.

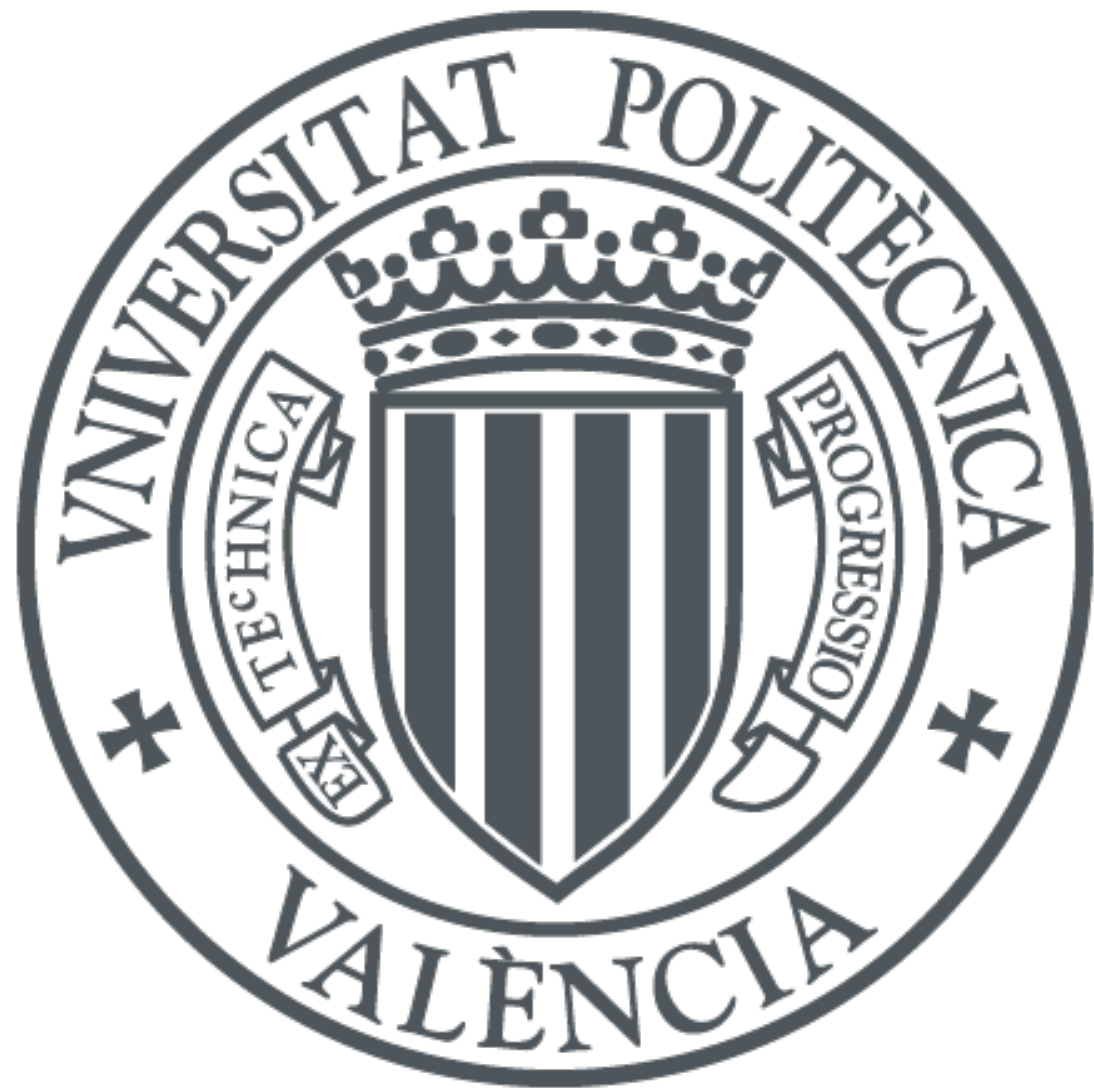

The final publication is available at

https://doi.org/10.1016/j.endm.2016.09.058

Copyright Elsevier

Additional Information

"NOTICE: this is the author's version of a work that was accepted for publication in Electronic Notes in Discrete Mathematics. Changes resulting from the publishing process, such as peer review, editing, corrections, structural formatting, and other quality control mechanisms may not be reflected in this document. Changes may have been made to this work since it was submitted for publication. A definitive version was subsequently published in Electronic Notes in Discrete Mathematics, [Volume 54, October 2016, Pages 337-342] DOI 10.1016/j.endm.2016.09.058 


\title{
Natural visibility graphs for diagnosing attention deficit hyperactivity disorder (ADHD)
}

\author{
Ainara Mira-Iglesias \\ Fundación para el Fomento de la Investigación Sanitaria y Biomédica \\ de la Comunitat Valenciana (FISABIO), Valencia, Spain \\ J. Alberto Conejero \\ Instituto Universitario de Matemática Pura y Aplicada \\ Universitat Politècnica de València, Spain \\ Esperanza Navarro-Pardo \\ Departamento de Psicología Evolutiva y de la Educación \\ Universitat de València, Spain
}

\begin{abstract}
Reaction times are described as a measure of perception, decision making, and other cognitive processes. For each individual, they usually follow an ex-gaussian distribution. However, this approach omits relationships between consecutive answers to tasks geared to evaluate attention. We show how natural visibility graphs (NVG's) can provide a further insight for analyzing these times and in the prediction of attention deficit hyperactivity disorder (ADHD) among young students.
\end{abstract}

Keywords: Natural visibility graph; attention deficit hyperactivity disorder; Kolmogorov-Smirnov test 


\section{Introduction}

Attention deficit hyperactivity disorder (ADHD) is a mental disorder that carries problems in paying attention, excessive activity, or difficulties for controlling behavior. In school-aged children, ADHD may provoke a poor academic performance. Its diagnosis is confirmed in the basis of psychiatric interviews received by the children, their parents, and their teachers. However, there is usually a backlog between the appearance of symptoms and the diagnosis and beginning of the treatment [7].

The reaction time (RT) is defined as the elapsed time between the presentation of a sensory stimulus and a subsequent behavioral response. It is understood as a measure of the processing speed. Alternative conductual approaches to the diagnosis of ADHD have been recently based on the modeling of RT's. Increased intra-individual variability in RT's is one highly consistent abnormalities in ADHD. Usually, reaction times follow an ex-Gaussian distribution. The fitting of the distribution of RT's to probabilistic functions is usually performed trying to maximize the likelihood estimation [9]. Several studies have been recently developed with the goal of setting a correspondence between the three parameters $(\mu, \sigma, \tau)$ of an ex-Gaussian distribution of RT derived from performance tests, such as the one of Conners' continuous performance tests [1], with mental disorders related to attention . In the last years, the more interesting parameter has been $\tau$ since it has been assumed to contain a perceptual portion of an RT, a decision component and it has been recently related to factors related to attention. For a recent account on the relevance of these three parameters in the diagnosis of ADHD, we refer to $[2,3]$. We point out, that these approaches cannot replace professional diagnosis of a mental disorder, however they permit to conduct wider screenings along large populations with a limited cost and it can permit to act when the first symptoms appear.

The natural visibility graph (NVG) introduced in [5] permits to give a visual representation of time series. These graphs inherit several properties of the underlying time series. For instance, periodic series are represented by regular graphs. In the same way, random series are represented by random graphs. Furthermore, there is a connection between fractal series and scalefree networks. As a matter of fact, stochastic time series are transformed into graphs whose degree distributions $P(k)$ follows a power-law function $\alpha k^{-\gamma}$ [6]. NVG's are also intended for deducing properties of the time series with the aid

1 Email: amisqrt2@gmail.com, aconejero@upv.es, esperanza-navarro@uv.es

2 The second author is partially supported by MEC project MTM2013-47093-P. 
of network science results. Visibility graphs have been used for representing complex time series that appear in different phenomena ranging from chaotic signals, heartbeat variability, economics, language structure, and seismology.

An NVG comes from the representation of the data of a time series on the plane. Let $\left(t_{1}, y_{1}\right),\left(t_{2}, y_{2}\right), \ldots,\left(t_{n}, y_{n}\right)$ be the observations of a time series, where $t_{i}$ holds for the time and $y_{i}$ for the magnitude measured. Each observation $\left(t_{i}, y_{i}\right)$ is represented by a segment from the point $\left(t_{i}, 0\right)$ to $\left(t_{i}, y_{i}\right)$, for all $1 \leq i \leq n$. The associated NVG graph $G=(V, E)$ is constructed as follows: Each one of these observations $\left(t_{i}, y_{i}\right)$ is in correspondence with a node $v_{i} \in V$ in the graph $G$. Two nodes $v_{i}, v_{j} \in V$ of $G$ are connected by an edge in $E$ if, and only if, we can connect the points $\left(t_{i}, v_{i}\right)$ with $\left(t_{j}, v_{j}\right)$ without crossing any segment joining $\left(t_{k}, 0\right)$ to $\left(t_{k}, y_{k}\right)$ for any $1 \leq k \leq n, k \neq i, j$. NVG's are connected, undirected, and invariant under affine transformations of the series data. For a detailed description of their properties we refer to [5].

In this work, NVG's are used as a tool to study the answers of students to a test of attention evaluation in order to detect which students are potential candidates of having an ADHD disorder. We show how the preliminary results obtained of a screening conducted over a group of students are congruent with the medical diagnosis of the mental disorders of them.

\section{Method and materials}

The experiment was carried out in a group of 38 students who were 8 years old and come from the Valencia region (Spain). The students took a lexical decision task test of type yes/no, processed under a DMDX software on a laptop. The test consists on presenting a set of letters (maybe only one) on the screen and then the student has to answer whether the letter $P$ appears or not there. This task is inspired in the adaptation of attention tasks for children presented in [8]. A total of 120 stimuli were presented to each student. A maximum time to answer to each item was set to $2.500 \mathrm{~ms}$. If the time expires $2.500 \mathrm{~ms}$. is assigned as answer time and a new stimulus is presented to the student. Answers faster than $100 \mathrm{~ms}$. were discarded.

For analyzing the results from the perspective of time series, we have just considered the time needed to answer by each student. If the answer was tight, then the time is considered as a positive magnitude. If not, we consider it with negative value. Since NVG's are invariant under affine transformations, we have added to all the values 2 times the lowest negative amount. This transformation permits us to consider all magnitudes as positive. 


\section{Results}

We first have studied the distribution of the degrees of the nodes of the visibility graph associated to each participant. Figure 1 illustrates the probability of a certain degree in the visibility graph for all the students of 8 year that took part in the study. The degrees of the NVG of each participant are represented by a different colour. At first sight, one can see that the results for 2 students are significantly different respect to the others, with a higher frequency of nodes of high order.

Afterwards, we have tried to verify whether the frequency of the degrees of the NVG's follow a power-law pattern. For instance, Figures 2 and 3 show the probabilities of the degrees of the NVG for a pair of students and its fitting to a power law function. Figure 2 corresponds to a student presenting some attention disorder and Figure 3 to a regular student. We can see how different is the behavior in both cases. The distribution of degrees in Figure 2 is concentrated in higher degrees. On the contrary, the probabilities for student 18, see Figure 3 are concentrated in lower degrees.

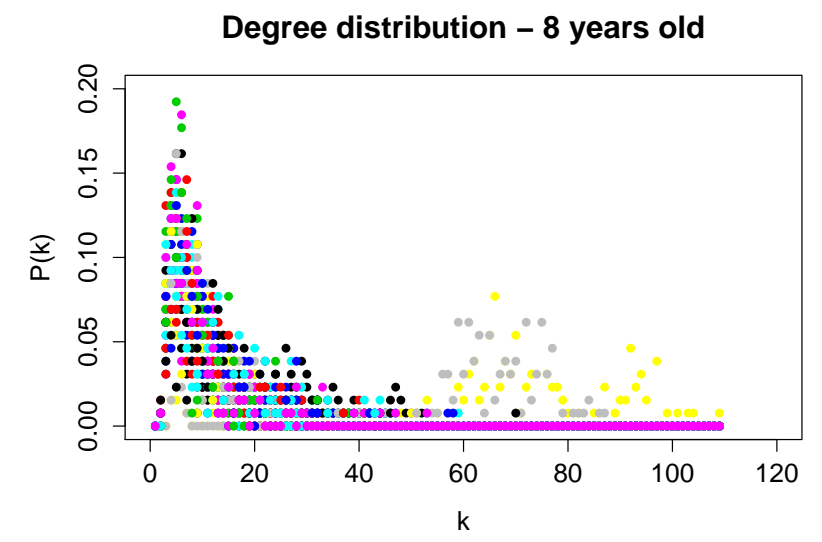

Fig. 1. Degree distribution of the visibility graphs of the participants.

In order to compare the different power law functions, we have computed the matrix of $p$-values from the application of the Kolmogorov-Smirnov (K-S) test to all pairs of participants in the study. This approach is quite similar to the one carried out in [4]. The results are presented in Figure 4. The powerlaw fitting of students 15, 16, and 17 differ considerably from the others, and its quite similar between them. We observe that at the $5 \%$ level of significance, we can reject the null hypothesis that any pair of students follow the same distribution. A careful look should also be paid to students 16 and 17 . 

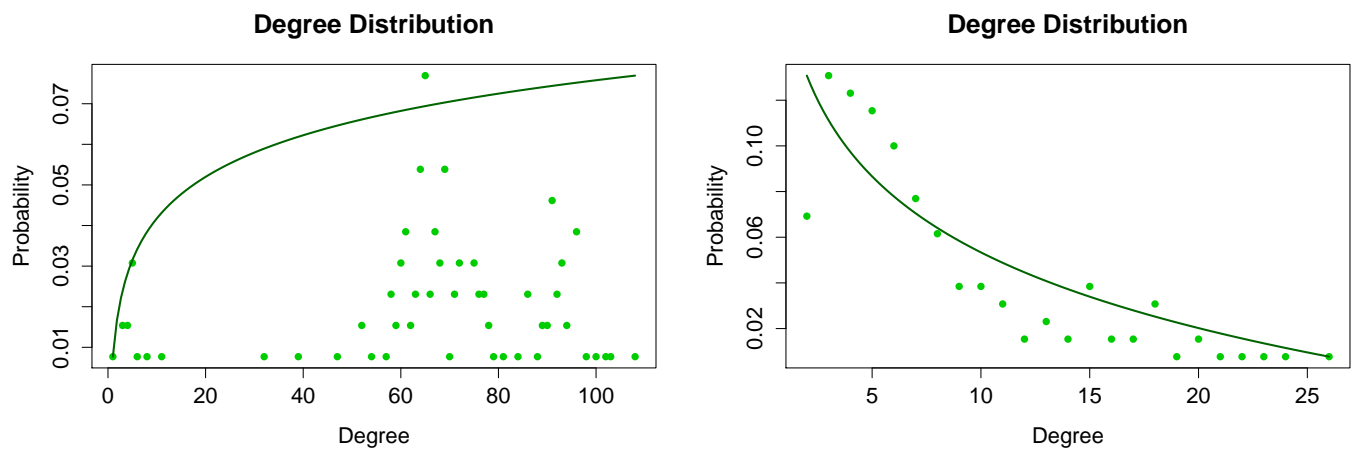

Fig. 2. Degree distribution (student 15). Fig. 3. Degree distribution (student 18).

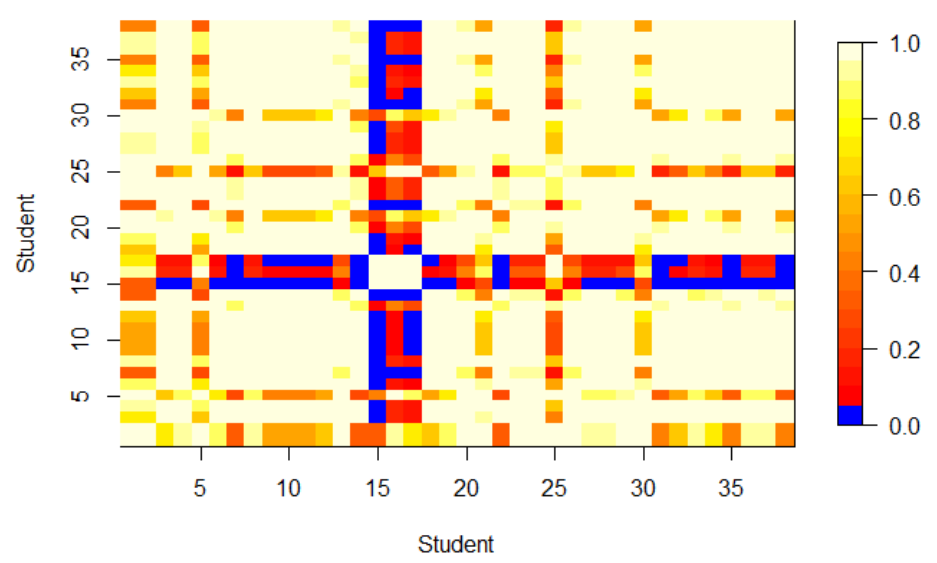

Fig. 4. Matrix of $p$-values from the application of the Kolmogorov-Smirnov (K-S).

\section{Discussion and conclusions}

After a clinical and independent clinical evaluation, these three students were diagnosed with ADHD and other behavioral disorders. One of them had a family history of ADHD and two of them were being medicated. The next applied objective of this research is to make a clinical diagnosis of subjects with no normative results in response times regarding their age group, in order to validate this detection methodology. We point out that these measurements cannot replace a clinical assessment, but it could help to make the correct diagnosis, mainly in a specially difficult field as ADHD[7]. 
To sum up, we have seen the potential use of this approach in screenings of ADHD. However, some improvements has still to be carried out. Our approach takes artificially whether the answers to the items were right or not. Other options can also be considered for treating the wrong answers. In fact, the three participants whose behavior differs from the others also show a significantly higher number of mistakes respect to the others. Furthermore, we have treated as equal missing responses and responses of $2500 \mathrm{~ms}$.

\section{References}

[1] C.K. Conners, J.N. Epstein, A. Angold, and J. Klaric. Continuous performance test performance in a normative epidemiological sample. Journal of Abnormal Child Psychology, 31:5 (2003) pp. 555-562.

[2] D. Gmehlin, A.B.M. Fuermaier, S. Walther, R. Debelak, M. Rentrop et al. Intraindividual variability in inhibitory function in qdults with ADHD - An ex-gaussian approach. PLoS ONE 9:12 (2014) e112298.

[3] S.H. Gu, S.S. Gau, S. Tzang, and W. Hsu. The ex-Gaussian distribution of reaction times in adolescents with attention-deficit/hyperactivity disorder, Research in Developmental Disabilities, 34:11 (2013) 3709-3719.

[4] L. Guzmán-Vargas, B. Obregón-Quintana, D. Aguilar-Velázquez, R. HernándezPérez, and L.S. Liebovitch. Word-length correlations and memory in large texts: a visibility network analysis. Entropy, 17:11 (2015) 7798-7810.

[5] L. Lacasa, B. Luque, F. Ballesteros, J. Luque, and J.C. Nuño. From time series to complex networks: The visibility graph. Proc. Natl. Acad. Sci. USA 105:13 (2008) 4972-4975.

[6] L. Lacasa, B. Luque, J. Luque, and J.C. Nuño. The visibility graph: A new method for estimating the Hurst exponent of fractional Brownian motion. Europhys. Lett. 86 (2009) 30001.

[7] E. Navarro-Pardo, J.C. Meléndez-Moral, A.S. Galán. Diagnóstico y tratamiento del trastorno por déficit de atención / hiperactividad: diferencias entre entrevista clínica y prueba psicométrica. Atención Primaria 44:1 (2012) 56-57.

[8] M.R. Rueda, M.K. Rothbart, B.D. McCandliss, L. Saccomanno, and M.I. Posner. Training, maturation, and genetic influences on the development of executive attention. Proc. Natl. Acad. Sci. USA 102:41 (2005) 14931-14936.

[9] Y. Lacouture and D. Cousineau. How to use MATLAB to fit the ex-Gaussian and other probability functions to a distribution of response times. Tutorials in Quantitative Methods for Psychology, 4:1 (2008) 35-45. 